\title{
Structural Health Monitoring Sensor Placement Optimization Under Uncertainty
}

\author{
Robert F. Guratzsch ${ }^{1}$ and Sankaran Mahadevan ${ }^{2}$ \\ Vanderbilt University, Nashville, TN, 37235
}

This paper develops a methodology for the optimum layout design of sensor arrays of structural health monitoring (SHM) systems under uncertainty. This includes finite element analysis under transient mechanical and thermal loads and incorporation of uncertainty quantification methods. The finite element model is validated with experimental data, accounting for uncertainties in experimental measurements and model predictions. The SHM sensors need to be placed optimally in order to detect with high reliability any structural damage before it turns critical. The proposed methodology achieves this objective by combining probabilistic finite element analysis, structural damage detection algorithms, and reliability-based optimization concepts.

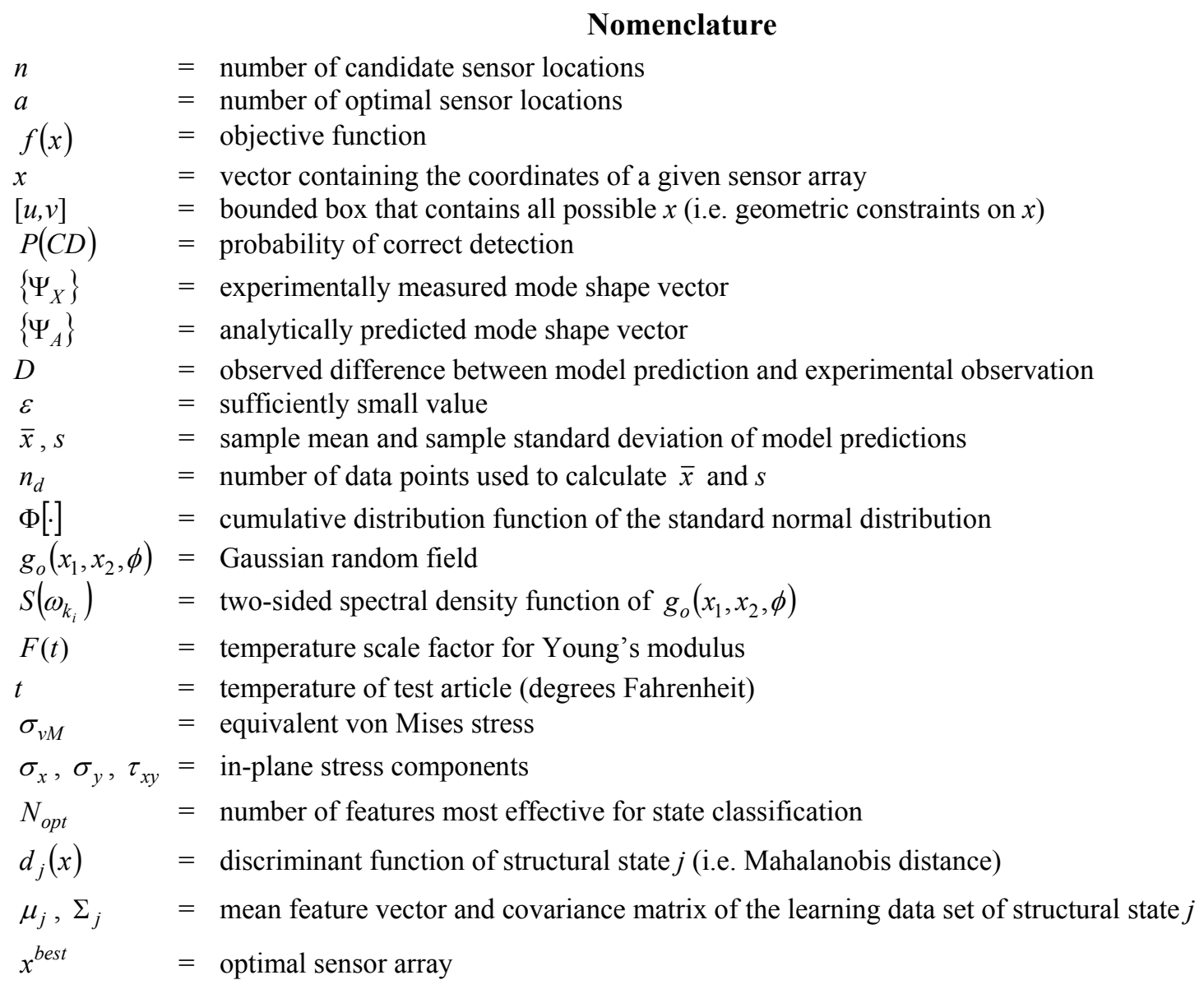

\footnotetext{
${ }^{1}$ Graduate Student, Civil \& Environmental Engineering Department, VU Station B 351831, 2301 Vanderbilt Place, Member (Student Upgrade).

${ }^{2}$ Professor of Civil \& Environmental Engineering, Civil \& Environmental Engineering Department, VU Station B 351831, 2301 Vanderbilt Place, AIAA Senior Member.
} 


\section{Introduction}

Structural health monitoring (SHM) systems that report in real-time the flight vehicle's condition in terms of reactions, stresses, and displacements, are central to meeting the demanding goals of increasing flight vehicle safety and reliability, while reducing vehicle operating and maintenance costs ${ }^{1}$. The SHM system must be small, lightweight, energy efficient, and the most reliable sub-system on board the flight structure in order to make incorporation into existing flight vehicle designs possible with minimal impact on the structure's performance. The structural behavior of next generation flight vehicles, such as the Space Operations Vehicle (SOV), is inherently random due to the uncertainties in the flight environment. A probabilistic structural analysis that includes the uncertainties associated with geometry, loads, and material properties is vital toward the success of the structural design. This includes the development of a finite element model, uncertainty quantification methods, and optimization techniques. Additionally, in order for the SHM system to detect with maximum probability any structural damage before it becomes critical, SHM system sensors need to be optimally placed. While many advances have been made in terms of sensor technology, damage detection algorithms, structural reliability, and deterministic sensor placement optimization (SPO) schemes, much additional research needs to be focused on probabilistic modeling, probabilistic analysis and design, as well as on SPO under uncertainty, in order to extract the maximum information about the structure's condition while taking the uncertainties into account.

This paper develops a methodology for integrating the advances in various individual disciplines for the optimum design of SHM system sensor arrays under uncertainty. The methodology aims at maximizing the probability of detecting damage by designing the locations of SHM system sensors. This includes the following steps: (1) structural simulation and model validation, (2) probabilistic analysis, (3) damage detection, and (4) sensor placement optimization. Section 2 of this paper defines the general methodology, while section 3 provides a numerical example to demonstrate SPO under uncertainty.

\section{Literature Review}

Several studies have investigated sensor placement optimization during recent years. Hiramoto, et al, ${ }^{2}$ as well as Abdullah, et al, ${ }^{3}$ have addressed the need to place actuators in an optimal way to control the behavior of dynamic structures, where Hiramoto, et al, uses the explicit solution of the algebraic Riccati equation to determine the optimal actuator placement and Abdullah, et al, utilizes genetic algorithms to solve the optimization. Genetic algorithms (GA) have also been employed to search for optimal locations of actuators in active vibration 
control. $^{4,5,6,7}$ With respect to SHM, Guo, et al, ${ }^{8}$ use a GA approach and a sensor placement optimization performance index based on damage detection to search for an optimal sensor array and Spanache, et al, ${ }^{9}$ use GA and account for economic/cost issues in the design of a cost optimal sensor system. However, GA-based sensor/actuator placement optimization methods often generate invalid strings during the evolution process and require a predefined number of discrete sensor configurations, which do not guarantee global optima.

Additionally, terrorism concerns have recently caused an increased interest in using sensor arrays for monitoring potential attacks on municipal water distribution systems. ${ }^{10,11,12}$ In this context, SPO has been attempted with respect to different objectives: 1) population exposed, ${ }^{13}$ 2) volume of contaminated water consumed, ${ }^{14}$ and 3) time to detection. ${ }^{15}$ However, Watson, et $a{ }^{16}{ }^{16}$ points out that in practice a multi-objective optimization, which simultaneously considers multiple performance measures is more appropriate. Other research areas that have shown a need for sensor networks and SPO include environmental monitoring (algae biomass monitoring, light intensity monitoring, etc.), ${ }^{17,18}$ monitoring of spatial distributions, ${ }^{19}$ and military surveillance operations ${ }^{20}$. In all of these methodologies, a predefined number of discrete sensor locations from which the sensor networks grow must be provided.

Related more closely to SPO of SHM systems of next generation flight vehicles, $\mathrm{Li}$, et al, ${ }^{21}$ proposed an algorithm that aims to identify modal frequencies and mode shapes best, as well as increase the signal to noise ratio. However, it is not shown that a sensor array that best identifies modal frequencies and mode shapes optimizes more traditional SHM performance measures such as the probability of correct classification. Gao and Rose $\mathrm{e}^{2223}$ define a probabilistic SPO approach, where a probabilistic damage detection model that describes detection probabilities over a confident monitoring region with radius $R$ is defined for each sensor of a given sensor set. The entire effectiveness of the sensor network is then assumed to be the joint effect of all sensors as estimated at a point by the union probability of all sensors. A covariance matrix adaptation evolution strategy is used to search the decision variable domain. Difficulties arise defining the probabilistic damage detection models and sources for uncertainty are not identified specifically. A similar SPO framework that addresses imprecise detection probabilities as well as uncertain terrain properties is proposed by Dhillon, et al. ${ }^{24}$ Parker, et al, ${ }^{25}$ address SPO for SHM based upon the concept of observability from the fields of dynamic systems theory and engineering design optimization. The technique uses a dynamical model of the structure in question to obtain performance measures with respect to damage detection and localization; however, it does not include uncertainty. 
To the authors' best knowledge the issues associated with SPO under uncertainty for SHM systems due to the spatial and temporal stochastic variability of material, geometric, and loading parameters have not been sufficiently addressed. The methodology developed in this paper includes the stochastic nature of the model input parameters to perform a probabilistic finite element analysis utilizing Monte Carlo realizations to derive the stochastic characteristics of the model outputs, which are used with appropriate damage detection algorithms to estimate probabilistic performance measures of a given sensor layout. Single-objective and multi-objective objective functions that utilize the probabilistic performance measures individually and in combination are considered. Additionally, a non-GA-based optimization algorithm, which considers the decision variables to be continuous and combines global and local searches, is employed.

\section{Proposed Methodology}

\section{A. Structural Simulation and Model Validation}

For most realistic structures, the response due to various loads cannot be determined via a closed-form function of the input variables. The response must be computed through numerical procedures such as a finite element method (FEM). Several finite element software packages are available. Regardless of the software package used, structural models and their corresponding simulations must capture all physical phenomena and include all relevant input parameters. The appropriate analysis may include linear, nonlinear, and/or coupled structural-thermal simulations.

In addition, model verification and validation is of extreme importance before employing the model results for damage detection and sensor layout optimization. Several validation metrics have been proposed to asses the predictive capability of models, such as the modal assurance criterion (MAC), the modal scale factor (MSF) ${ }^{26}$ and a newly developed model reliability metric $(\mathrm{MRM})^{27}$. Validation of numerical models by comparison against experimental observations has to account for errors and uncertainties in both model predictions and measured observations. MRM accounts for the probabilistic nature of and the uncertainty associated with both model predictions and laboratory observations (i.e. standard deviations, distributions, etc.). 

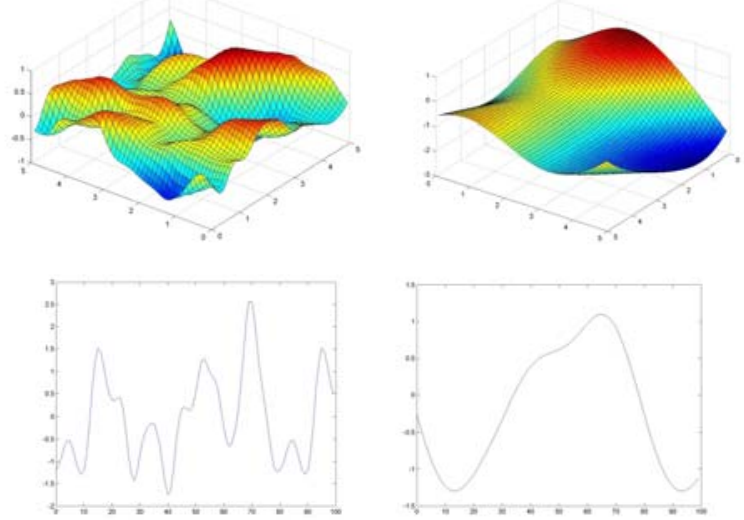

Figure 1. Random field and process realizations with varying correlation structures.

\section{B. Probabilistic Analysis}

Structural model parameters such as distributed loads

and material and geometric properties have temporal and spatial variability and cannot be expressed as single random variables, but must be represented as random processes and random fields ${ }^{28}$. Thus random process/field modeling is a key step in probabilistic finite element analysis. The Karhunen-Loeve Expansion (KLE) has been used extensively to simulate Gaussian random processes. ${ }^{29}$ The wavelet

transform method is an extension of the KLE simulation algorithm and is applicable to non-stationary Gaussian processes and fields. Other random process/field generation sequences include the Pierson-Moskowitz Wave Spectra, the JONSWAP spectra ${ }^{30}$, Sakamoto's Polynomial Chaos Decomposition ${ }^{31}$, and Shinozuka's Gaussian stochastic process formulation ${ }^{32}$. For example, the Gaussian stochastic fields and processes in Figure 1 were generated using Shinozuka's spectral representation formulation and the Wiener-Khinchine relations. Random field realizations, such as the ones shown can be used to simulate component thickness, material moduli, and spatially distributed loads such as thermal and pressure loading. Representing spatially or temporally distributed model inputs through discretized random process/field realizations allows the inclusion of their uncertainty in FEM analyses.

Once the model input parameters are randomly generated via the discretization of random processes/fields and applied as inputs to FEM models, repeated simulations of the finite element analysis at each realization are used to generate statistical and/or sensitivity information on model outputs at each possible sensor location $i$. For practical purposes, each node of the FEM model may represent a possible sensor location.

\section{Damage Detection Algorithms}

Damage detection and location identification algorithms include wavelet-based approaches ${ }^{33}$, two-stage modal frequency analysis ${ }^{34}$, and methods for eddy-current-based damage detection ${ }^{35}$. Property matrix updating, nonlinear response analysis, and damage detection using neural networks are all methods used to manipulate the information gathered by SHM systems for decision making. However, most structural damage detection methods and algorithms found in the literature examine the changes in the measured structural vibration response and analyze the modal 
frequencies, mode shapes, and flexibility/stiffness coefficients of the structure ${ }^{36}$. This can be achieved either actively or passively, where active damage detection algorithms utilize the system response to an auxiliary excitation and passive methodologies utilize only the responses to operational vibrations. A comprehensive review of the state of the art damage detection and location identification algorithms is provided in Ref. 36.

The probabilistic FEM analysis in the previous section quantifies the statistics of the model outputs at all possible sensor locations. Additional analysis is needed to estimate the probability of correctly identifying the structural state of a component for a given sensor layout, $x$ (i.e. $P(C D)=P($ correct structural classification $\mid$ sensor layout $x)$ ). This can be accomplished via any appropriate diagnostics signal analysis procedure (i.e. damage detection algorithm). The signal analysis procedure employed in this study follows the general concepts of Ref. 37 and utilizes the feature extraction and state classification methodologies defined in Ref. 38. Repeated analyses using different realizations of the random inputs to healthy and damaged structural FEM models and their respective state classification constructs a classification matrix from which several performance measures of the given sensor layout can be estimated. Further details of such a procedure are given in Section IV.C.

\section{Sensor Placement Optimization}

The SPO problem can be generalized as "given a set of $n$ candidate locations, find $a$ locations, where $a<<n$, which provide the best possible performance, ${ }^{39}$ in damage detection. Studies by Padula ${ }^{39,40,41}$ and Raich $^{42}$ have examined the problems and issues involved with SPO. Integer and combinatorial optimization methods have been used to optimize the placement of actuators for vibration control and noise attenuation. In addition, genetic algorithms for the optimization of sensor layouts ${ }^{42}$ have been proposed. Multivariate stochastic approximation using simultaneous perturbation gradient approximation allows for the inclusion of noise in function evaluations or experimental measurements and has been shown to be efficient for large-dimensional problems ${ }^{43}$.

An approach to SPO that includes uncertainty is to employ Snobfit ${ }^{44}$ (Stable Noisy Optimization by Branch and Fit), an optimization scheme that is designed for bound-constrained optimization of noisy objective functions, which are costly to evaluate due to computational or experimental complexity. The major advantage of using Snobfit is that the algorithm does not require a previously determined set of candidate sensor locations, but rather considers the following optimization problem. 


$$
\begin{aligned}
& \min f(x) \\
& \text { s.t. } x \in[u, v]
\end{aligned}
$$

where $x$ is continuous and $[u, v]$ is a bounded box in $\Re^{n}$ with a nonempty interior. ${ }^{44}$

The underlying idea of the optimization formulation is to identify a sensor layout, $x$, that will maximize some performance measure, such as the probability of correctly classifying the structure as either healthy or damaged (i.e. classify the structure as healthy when it is indeed healthy and as damaged when it is damaged). Here $x$ represents a vector containing the coordinates of the SHM sensors for a given layout. From the reliability analysis described above and a diagnostics signal analysis procedure, a performance measure such as $P(C D)$, is known. This allows the optimization formulation given in Eq. (1) to be utilized, where $f(x)=-P(C D)$ and $[u, v]$ are the geometric constraints on $x$ given by the physical dimensions of the structure.

\section{Application of Methodology}

The proposed methodology is implemented using the following example problem. The structure under consideration is a simplified thermal protection system (TPS) component that is described in detail in Ref. 45, and shown in Figure 2. The test article consists of a heat-resistant, 0.25 inch thick aluminum plate, held in place via four 0.25 inch diameter bolts located 0.50 inches from the edges of the plate.

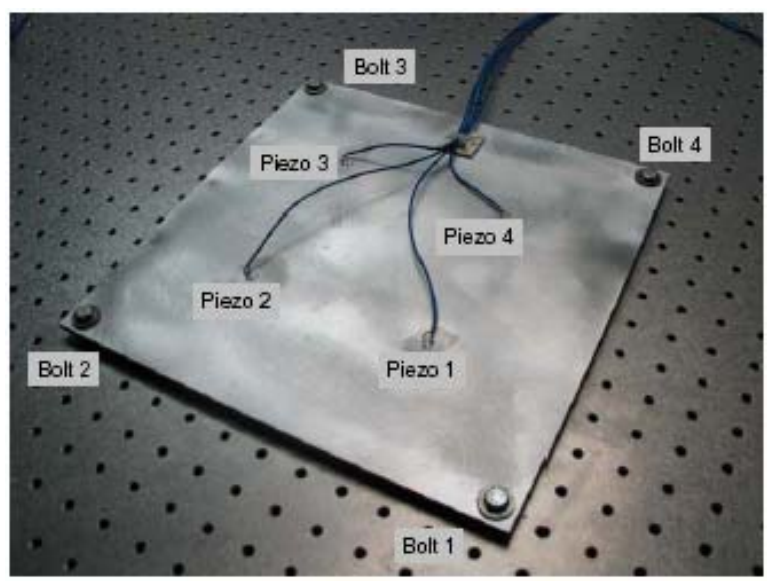

Figure 2. Experimental setup of TPS test article showing bolts and piezoelectric transducer placement [45].

\section{A. Structural Simulation and Model Validation}

The structure under consideration is modeled using the commercial finite element software Ansys. ${ }^{46}$ A portion of the FEM model is shown in Figure 3. Four-noded shell elements (Shell63) and two-noded spring elements (Combin14) are utilized to model the aluminum plate and bolted boundary conditions. Approximately 3,300 nodes and 2,800 elements comprise the 19,836 degree of freedom (DOF) models. In Figure 3, the four points located near the corners of the plate simulate the bolted boundary conditions via 48 spring elements per bolt with varying stiffness coefficients (depending on which structural state the model simulates), while the point near the center of the upper left quadrant of the plate simulates the piezoelectric actuator. The analysis is transient and 
includes a dynamic mechanical load consisting of a sinusoidal frequency sweep, exciting the structure from 0 to $1500 \mathrm{~Hz}$ in approximately 2.0 seconds. This excitation represents the auxiliary input used with active damage detection algorithms. Due to the high frequency of the excitation function, a mode superposition (MSP) transient analysis was used to evaluate the FEM model simulations. MSP analysis sums factored mode shapes, obtained from a modal analysis, to calculate the dynamic response ${ }^{46}$. MSP assumes that the structure behaves linearly.

This study employs two metrics for validation of the finite element models. The modal assurance criterion $(\mathrm{MAC})^{26}$ provides a measure of the statistical correlation between model predictions and experimental observations.

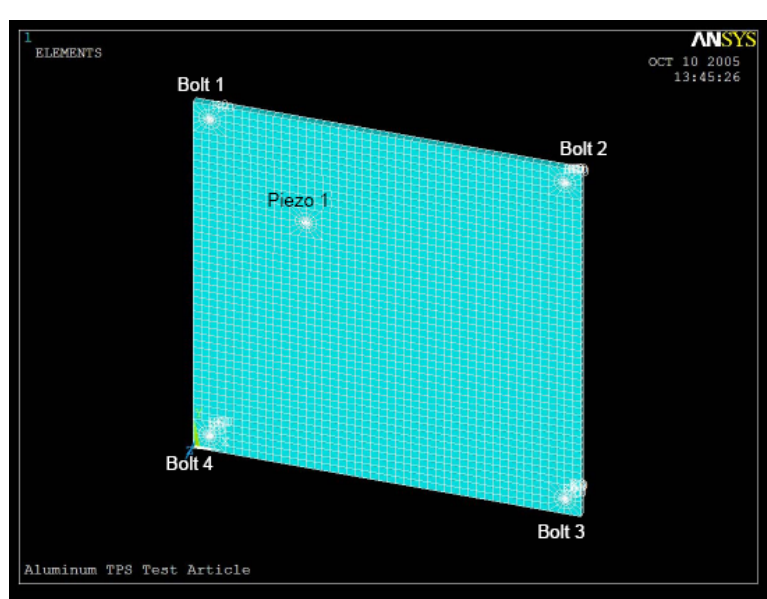

Figure 3. Finite element model of TPS component.
$\left\{\Psi_{X}\right\}$ defines the experimentally measured mode shape vector and $\left\{\Psi_{A}\right\}$ the analytically predicted mode shape vector. MAC is defined as

$$
\frac{\left.\mid\left\{\Psi_{X}\right\}^{T} \cdot\left\{\Psi_{A}\right\}\right\}^{2}}{\left(\left\{\Psi_{X}\right\}^{T} \cdot\left\{\Psi_{X}\right\}\right)\left(\left\{\Psi_{A}\right\}^{T} \cdot\left\{\Psi_{A}\right\}\right)}
$$

and is a scalar quantity close to 1.0 if the experimental and theoretical mode shapes are in fact from the same mode. If the two mode shapes, which are being compared, actually relate to two different modes of vibration, a value close to

0.0 should be obtained. Generally speaking, a value in excess of 0.9 implies well correlated modes ${ }^{26}$.

In addition to MAC, Ref. 27 defines a reliability measure via the simple metric $r=P(-\varepsilon<D<\varepsilon)$, i.e. the probability that the observed difference, $D$, between model prediction and experimental observation is less than a small value $\varepsilon$. It is calculated as

$$
r=\Phi\left[\frac{\sqrt{n_{d}}(\varepsilon-|\bar{x}-\Theta|)}{s}\right]-\Phi\left[\frac{\sqrt{n}_{d}(-\varepsilon-|\bar{x}-\Theta|)}{s}\right],
$$

where $\Theta$ is a single-valued experimentally observed measurement, $\bar{x}$ is the sample mean and $s$ the sample standard deviation of the model predictions, and $n_{d}$ is the number of data points utilized to calculate $\bar{x}$ and $s$. The cumulative distribution function (CDF) of the standard normal distribution is denoted as $\Phi[\cdot]$. In addition to comparing modal frequencies via MRM, mode shape vectors may also be investigated. These comparisons and their results ${ }^{47}$ lead to the conclusion that all model predictions are highly correlated to experimental observations in 
regards to natural frequencies as well as mode shapes, such that the models can be considered validated with high reliability.

\section{B. Probabilistic FEM Analysis}

In the current example, plate thickness, Young's modulus, Poison's ratio, and density are modeled as Gaussian random fields with independent, but equal correlation structures along orthogonal axes. A two-dimensional stochastic process was generated for these model inputs using the spectral representation as defined in Eq. (4) via Shinozuka's formulation ${ }^{48}$ and the Wiener-Khinchine relations ${ }^{49}$. The Gaussian random field $g_{o}\left(x_{1}, x_{2}, \phi\right)$ can be simulated by the following series as $N_{1}$ and $N_{2}$ approach infinity.

$$
g_{o}\left(x_{1}, x_{2}, \phi\right)=2 \sum_{k_{1}=1}^{N_{1}-1} \sum_{k_{2}=1}^{N_{2}-1}\left[\sqrt{S\left(\omega_{k_{1}}\right) S\left(\omega_{k_{2}}\right) \Delta \omega_{1} \Delta \omega_{2}} \cos \left(\omega_{k_{1}} x_{1}+\omega_{k_{2}} x_{2}+\phi_{k_{1}, k_{2}}\right)\right]
$$

where $\Delta \omega_{i}=\omega_{u_{i}} / N_{i}, \omega_{k_{i}}=k_{i} \Delta \omega_{i}$, for $i=1,2$. Here $\omega_{u_{i}}$ is the upper cutoff frequency beyond which $S\left(\omega_{k_{i}}\right)$ is considered zero. $S\left(\omega_{k_{i}}\right)$ is the two-sided power spectral density function of the random field in the $i$ direction and $\phi_{k_{1}, k_{2}}$ an array containing the independent random phase angles uniformly distributed between 0 and $2 \pi . N_{i}$ defines the number of terms to be included in the dual summation in the $i$ direction. The random fields in this study utilize the following power spectral density functions: $S\left(\omega_{k_{i}}\right)=1 / 4 \sigma_{i}^{2} b_{i}^{3} \omega_{k_{i}}^{2} \cdot \exp \left(-b_{i} \omega_{k_{i}}\right)$ for $i=1,2$. Here $\sigma_{i}$ is the standard deviation of the stochastic process in the $i$ direction and $b_{i}$ its corresponding "correlation distance."

Table I. Mean and COV values used for random field simulation.

\begin{tabular}{|c|c|c|c|c|}
\hline & $\begin{array}{c}\text { Panel Thickness } \\
\text { (in) }\end{array}$ & $\begin{array}{c}\text { Young's Modulus } \\
\text { (psi) }\end{array}$ & $\begin{array}{c}\text { Poison's } \\
\text { Ratio }\end{array}$ & $\begin{array}{c}\text { Density } \\
\text { (lb-mass/in^3) }\end{array}$ \\
\hline Mean & 0.2458 & $9.75 \mathrm{E}+06$ & 0.3 & $2.59 \mathrm{E}-04$ \\
\hline COV & 0.02 & 0.02 & 0.02 & 0.02 \\
\hline
\end{tabular}

For the random fields considered as FEM inputs to models of the test article, $b_{1}=b_{2}=3$ and $\sigma_{1}=\sigma_{2}=1$, where the magnitude of $g_{o}\left(x_{1}, x_{2}, \phi\right)$ is scaled after the fact to match the mean and coefficient of variation $(\mathrm{COV})$ of the random field to be simulated. $\omega_{u_{1}}=\omega_{u_{2}}=5 \pi$, while $N_{1}=N_{2}=35$. Table I lists the means and COV used for each of the random fields simulated with Eq. (4).

Temperature uncertainty was included as a random variable uniformly distributed between 65 and 75 degrees Fahrenheit. The following temperature effect model was constructed via a quadratic regression analysis of data in Ref. 50: 


$$
F(t)=\left(-1.151525 \times 10^{-6}\right) t^{2}+\left(2.75775 \times 10^{-5}\right) t+1.00067
$$

where $F(t)$ is a scale factor for Young's modulus and $t$ is the plate temperature in degrees Fahrenheit.

Repeatedly executing deterministic finite element analyses using realizations of the model inputs provides data for statistical analysis of the model responses. For the example at hand, 500 simulations using 500 realizations of the random inputs were executed; 100 simulations of the healthy model, 100 simulations of the model damaged at bolt 1, 100 simulations of the model damaged at bold 2, and so on, where a damaged bolt refers to a bolt at $25 \%$ nominal torque (damage was simulated analytically by altering the stiffness constants of the spring elements surrounding each bolt location). These 5 sets of simulations and their corresponding response statistics are used for damage detection.

\section{Damage Detection and State Classification}

Figure 4 shows a typical sensor layout, where sensor location 1 is the point of input excitation and stationary, while sensor locations 2, 3, and 4 are the points of sensing and variable. Also shown in Figure 4 are the locations of the 4 bolts which hold the test structure in place and are the locations of fastener damage. The hatched areas in Figure 4 are regions where it is infeasible to place SHM sensors.

From the pool of simulation output of the probabilistic FEM analysis consisting of temporal displacement data, an equivalenced von Mises stress is calculated via Eq. (6) as defined in Ref. 46 for each possible sensor location.

$$
\sigma_{v M}=\sqrt{\sigma_{x}^{2}-\sigma_{x} \sigma_{y}+\sigma_{y}^{2}+3 \tau_{x y}^{2}}
$$

where $\sigma_{x}, \sigma_{y}$, and $\tau_{x y}$ are the in-plane stress components, which are estimated from the displacement records of the four nearest neighboring nodes. Plane stress conditions are assumed. 


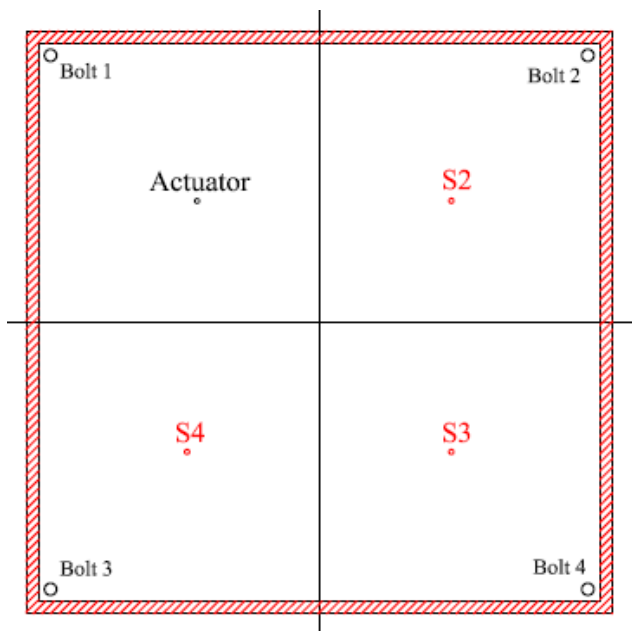

Figure 4. - TPS Test Plate with Typical Sensor Layout, Actuator, and Fastener Damage Locations.
From the estimated von Mises stress records at sensor locations S2, S3, and S4, a set of features is extracted. Features are characteristics unique to a signal generated under a given set of parameters. The set of features utilized for this example problem is based in the frequency-domain and is extracted via the well-known Welch method ${ }^{51,52}$ from the power spectral densities (PSD) of the signals. With a bin size of 100 measurements and an overlap of $50 \%$, the Welch method produces 51 features each from the signals obtained at piezoelectric sensor locations S2, S3, and S4. Dimensionality reduction is achieved via feature selection. Feature selection provides a subset of $N_{\text {opt }}$ features from the $m$-dimensional

feature pool most effective for state classification. In general, the fewer features used in a classifier, the more likely the training set performance will be representative of test set performance ${ }^{38}$. A sequential forward search algorithm ${ }^{53,54}$ is used to identify 25 optimal features from the original 153 -dimensional feature pool.

The above defined feature vector is then used for state classification. The state classifier utilized in this work is derived from Bayes decision theory and minimizes the probability of classification error ${ }^{37}$. The discriminant functions, one for each structural state ("healthy", "damaged at bolt 1", "damaged at bolt 2", etc.), are the Mahalanobis distances as given in Eq. (7).

$$
d_{j}(x)=\left(x-\mu_{j}\right)^{t} \Sigma_{j}^{-1}\left(x-\mu_{j}\right)
$$

where $j$ indexes the structural state, $x$ is a feature vector to be classified, and $\mu_{j}$ and $\Sigma_{j}$ are the mean feature vector and covariance matrix of the learning data set of structural state $j$. The learning data set consists of the first 50 simulations of each structural state. Since the Mahalanobis distance requires the determination of the inverse of $\Sigma_{j}$ it is necessary that the feature covariance matrix be non-singular. State classification is continued by evaluating each discriminant function for each simulation of the testing data set and assigning the state according to the discriminant function with the smallest value.

It has been shown in Ref. 55 that the above damage detection algorithm works most efficiently in comparison with damage detection algorithms which utilize other feature types, feature extraction methods, dimensionality 
reduction schemes, and feature selection algorithms. This damage detection algorithm is then applied to testing data, which consists of the second 50 simulations of each structural state. This yields a classification matrix corresponding to a given sensor layout, from which several performance measures may be estimated. A sample classification matrix is shown in Table II. Training and testing data sets are reversed to achieve higher fidelity within the classification matrix.

Table II. Sample classification matrix for a given sensor layout.

\begin{tabular}{|c|c|c|c|c|c|c|}
\hline \multicolumn{2}{|c|}{} & \multicolumn{5}{c|}{ Classified States } \\
\cline { 2 - 7 } & $\begin{array}{c}\text { Damaged } \\
1\end{array}$ & $\begin{array}{c}\text { Damaged } \\
2\end{array}$ & $\begin{array}{c}\text { Damaged } \\
3\end{array}$ & $\begin{array}{c}\text { Damaged } \\
4\end{array}$ & Healthy \\
\hline \multirow{4}{*}{$\begin{array}{c}\text { True } \\
\text { States }\end{array}$} & $\begin{array}{c}\text { Damaged } \\
1\end{array}$ & 78 & 21 & 0 & 0 & 1 \\
\cline { 2 - 7 } & $\begin{array}{c}\text { Damaged } \\
2\end{array}$ & 0 & 98 & 0 & 2 & 0 \\
\cline { 2 - 7 } & $\begin{array}{c}\text { Damaged } \\
\text { Damaged } \\
4\end{array}$ & 0 & 1 & 91 & 7 & 1 \\
\cline { 2 - 7 } & Healthy & 0 & 11 & 0 & 1 & 88 \\
\hline
\end{tabular}

Using the information contained in the classification matrix one can estimate several probabilistic performance measures of a given sensor layout, such as the probability of false alarm (Type I Error), the probability of missed detection (Type II Error), the probability of correct detection (Accuracy), and the probability of

misdetection (1-Accuracy $)^{56} . \quad P($ False Alarm) is defined as the likelihood that the damage detection algorithm classifies a healthy structure as damaged. $P$ (Missed Detection) is the probability that the damage detection method classifies a damaged structure as healthy. Accuracy is measured via $P$ (Correct Detection), which is defined as the probability that the damage detection method will classify a given structure correctly into its proper structural state (i.e. $P\left(\right.$ classify structure as $\omega_{i} \mid$ structural state is $\left.\omega_{i}\right)$ ). The compliment of $P($ Correct Detection) is $P($ Misdetection $)$. These probabilities can be used to evaluate a given sensor array. The performance measures are expressed as follows.

$$
\begin{gathered}
P(\text { False Alarm })=\frac{\text { Sum of First } 4 \text { Elements in Row } 5 \text { of } \mathrm{CM}}{\text { Sum of All Elements in Row } 5 \text { of } \mathrm{CM}}=P(\text { TypeI }) \\
P(\text { Missed Detection })=\frac{\text { Sum of First } 4 \text { Elements Column } 5 \text { of } \mathrm{CM}}{\text { Sum of All Elements in Column } 5 \text { of } \mathrm{CM}}=P(\text { TypeII }) \\
P(\text { Correct Detection })=\frac{\text { Sum of Diagonal Elements of } \mathrm{CM}}{\text { Sum of All Elements of CM }}=P(C D) \\
P(\text { Misdetection })=1-P(\text { Correct Detection })
\end{gathered}
$$


Evaluating Eqs. (8) to (11) for the classification matrix shown in Table II yields the following results. $P($ False Alarm $)=0.12 ; \quad P($ Missed Detection $)=0.033 ; \quad P($ Correct Detection $)=0.89 ; \quad P($ Misdetection $)=0.11$.

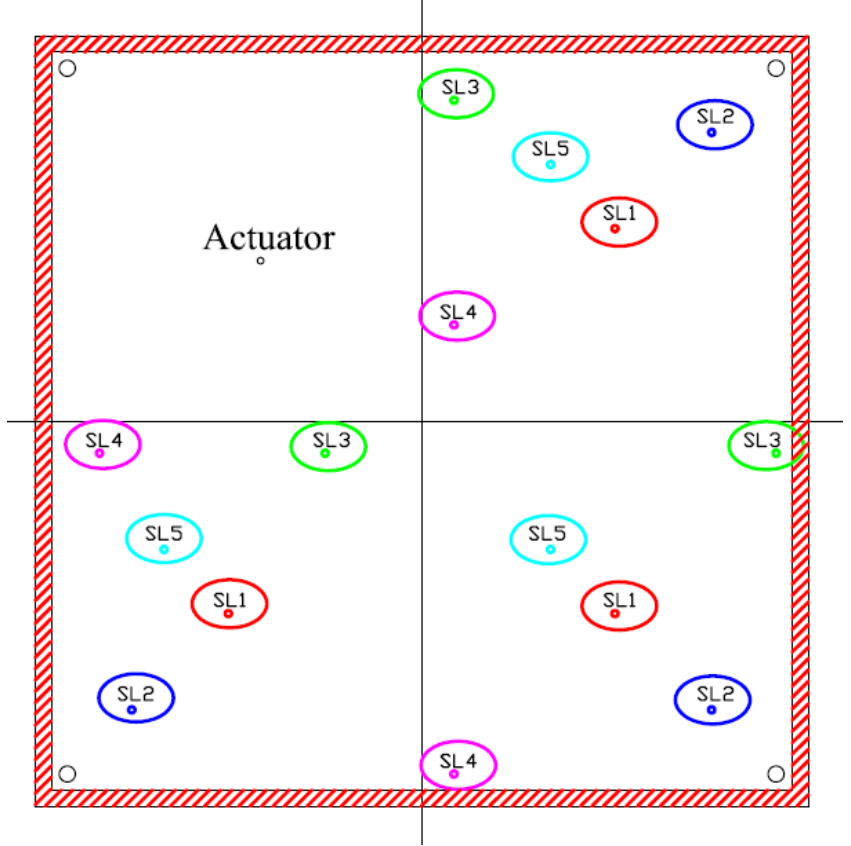

Figure 5. Five randomly selected sensor layouts.

Table III. Performance measures corresponding to randomly selected sensor layouts of Figure 5.

\begin{tabular}{|c|c|c|c|}
\hline $\begin{array}{c}\text { Sensor } \\
\text { Layout }\end{array}$ & $P(C D)$ & $P($ Type I) & $P($ Type II) \\
\hline SL1 & 0.916 & 0.12 & 0.0075 \\
\hline SL2 & 0.860 & 0.08 & 0.0050 \\
\hline SL3 & 0.866 & 0.12 & 0 \\
\hline SL4 & 0.894 & 0.11 & 0.0025 \\
\hline SL5 & 0.872 & 0.10 & 0.0075 \\
\hline
\end{tabular}

Repeating the above calculations for different sensor layouts generates different values of the performance measures. Five randomly selected sensor layouts are shown in Figure 5 and their corresponding performance measures are shown in Table III. Note that for the 5 sensor layouts presented, the best sensor layout varies depending on which performance measure is utilized. For example, if maximum $P(C D)$ is desired, sensor configuration SL1 is optimum; however, if minimizing $P($ TypeI $)$ is the objective, sensor layout SL2 performs best. A multi-objective optimization problem with an objective function of the form shown in Eq. 12 may be utilized.

$$
f(x)=\alpha \cdot P(C D)+\beta \cdot P(\text { TypeI })+\gamma \cdot P(\text { TypeII })(12)
$$

This however generates the additional problem of assigning values to $\alpha, \beta$, and $\gamma$, which may prove to be difficult and depending on what values are chosen may cause the optimal solution to vary significantly.

\section{Sensor Placement Optimization}

The software package Snobfit ${ }^{44}$, programmed in $\mathrm{Matlab}^{57}$, is used to solve the optimization formulation given by Eq. (1) iteratively. Snobfit is designed specifically to handle the following difficulties that arise with this particular problem. 
- The function values are expensive to evaluate (i.e. obtaining the performance measures for a given sensor layout is computationally intensive).

- Instead of a function value requested at a point $x$, only a function value at some nearby point $\tilde{x}$ is returned (the finite mesh size of the FEM models restricts that "sense-able" responses required by the damage detection algorithm to estimate the performance measures are only available at nodal locations).

- The function values are noisy (due to the finite number of simulations utilized to construct the confusion matrix, there is a finite precision with which the performance measures can be estimated).

- The objective function may have several local minima.

- Gradient information is not readily available.

The Snobfit algorithm proceeds as follows to solve Eq. (1). It partitions the bounded box $[u, v]$ into a set of subboxes such that each contains exactly one point. A branching algorithm is utilized for this purpose. Snobfit then builds local quadratic models, around the current best point, $x^{\text {best }}$, and around all other points. Two different types of quadratic models are used: a Hessian fit around the best point, $x^{\text {best }}$, and around all others a quadratic fit using as Hessian a suitable multiple of the Hessian matrix used in the model around $x^{\text {best }}$. The suitable multiple is decided based on the point's distance from $x^{\text {best }}$. The algorithm then suggests a user-specified number of evaluation points to be used in the next iteration of the optimization. ${ }^{44}$

The function can be evaluated at these points and other locations for further Snobfit iterations. Due to the fact that the structural FEM model described in Section IV.A. has a finite fidelity and temporal data is only available at the nodes, the points which Snobfit requests are substituted with the nearest neighboring nodal locations. The damage detection and state classification procedure described in Section IV.C. is performed for these requested sensor layouts, where vectors $u$ and $v$ confine sensors 2,3 , and 4 to their respective quadrants of the TPS component. A natural stopping criterion would be to quit exploration if for a number of iterations no new $x^{\text {best }}$ is generated and the accuracy of $x^{\text {best }}$ as predicted by Snobfit converges to a reasonably small value. 
Table IV. Results: optimal sensor arrays corresponding to different objective functions.

\begin{tabular}{|c|c|c|c|c|c|c|c|c|c|}
\hline \multirow{2}{*}{$\begin{array}{c}\text { Objective Function } \\
\mathrm{f}(\mathrm{y})=\end{array}$} & \multirow{2}{*}{ Nite } & \multirow{2}{*}{ Nobj } & \multicolumn{3}{|c|}{ Optimal Solution Coordinates for } & \multirow{2}{*}{$E$} & \multicolumn{3}{|c|}{$\begin{array}{c}\text { Corresponding Performance } \\
\text { Measures }\end{array}$} \\
\cline { 10 - 12 } & & & $\mathrm{S} 2$ & $\mathrm{~S} 3$ & $\mathrm{~S} 4$ & & $\mathrm{P}(\mathrm{CD})$ & $\mathrm{P}($ Type I) & $\mathrm{P}($ Type II) \\
\hline$-\mathrm{P}(\mathrm{CD})$ & 71 & 258 & $8.75,6.75$ & $6.0,3.5$ & $3.5,0.75$ & 0.0104 & 0.944 & 0.13 & 0.0075 \\
\hline $\mathrm{P}($ Type I) & 12 & 58 & $7.0,8.5$ & $11.73,0.27$ & $5.75,1.25$ & 0.0426 & 0.916 & 0.01 & 0 \\
\hline $\mathrm{P}($ Type II) & $\mathrm{n} / \mathrm{a}$ & $\mathrm{n} / \mathrm{a}$ & $7.0,8.5$ & $11.73,0.27$ & $5.75,1.25$ & $\mathrm{n} / \mathrm{a}$ & 0.916 & 0.01 & 0 \\
\hline$-0.5 \mathrm{P}(\mathrm{CD})+0.25 \mathrm{P}($ Type I)+0.25 P(Type II) & 55 & 268 & $6.75,8.75$ & $11.60,0.40$ & $5.75,1.25$ & 0.0208 & 0.932 & 0.03 & 0.0025 \\
\hline $0.5(1-\mathrm{P}(\mathrm{CD})++0.25 \mathrm{P}($ Type I)+5.0 P(Type II) & 44 & 196 & $7.0,8.5$ & $11.73,0.27$ & $5.75,1.25$ & 0.0375 & 0.916 & 0.01 & 0 \\
\hline
\end{tabular}

The above defined procedure was carried out for the objective functions listed in the first column of Table IV. The first three objective functions are single-objective. The $4^{\text {th }}$ and $5^{\text {th }}$ objective functions are of the form shown in Eq. 12, where $\alpha=-0.5, \beta=0.25$, and $\gamma=0.25$ for the $4^{\text {th }}$ objective function and $\alpha=-0.5, \beta=0.25$, and $\gamma=5.0$ for the $5^{\text {th }}$ objective function. In addition, the compliment of $P(C D), \quad[1-P(C D)]$, is utilized in combination with $\alpha$ as the first term of the $5^{\text {th }}$ objective function to adjust the relative importance of each of the three performance measures to a ratio of approximately $2: 1: 1$. This brings the three individual objectives to a comparable scale. The approximate ratio of relative importance of the three performance measures for the $4^{\text {th }}$ objective function is $360: 20: 1$. Other ratios of relative importance may be achieved by adjusting $\alpha, \beta$, and $\gamma$. The corresponding results are also shown in Table IV, where Nite is the number of Snobfit iterations, Nobj is the number of objective function evaluations, and $E$ is the measure of accuracy of the quadratic model at the optimal solution as estimated by Snobfit. The coordinates given are with respect to the bottom left corner of the plate. The results are visually presented in Figure 6.

From Table IV and Figure 6 it can concluded that although the solution varies for different objective functions, the optimal sensor arrays corresponding to objective functions 2 through 5 are virtually identical. Additionally, it was observed during Snobfit's iterations that the optimal solutions to objective functions 2 through 5 was robust and insensitive to small changes in the independent variables (i.e. shifting sensors S2, S3, and/or S4 by less than 0.25 inches in any direction, did not significantly alter the performance measures). However, the solution to the $1^{\text {st }}$ objective function, $f(x)=-P(C D)$, was very sensitive with respect to small changes in the independent variables (i.e. shifting sensors S2, S3, and/or S4 by less than 0.25 inches in any direction, significantly degraded the performance measures). 


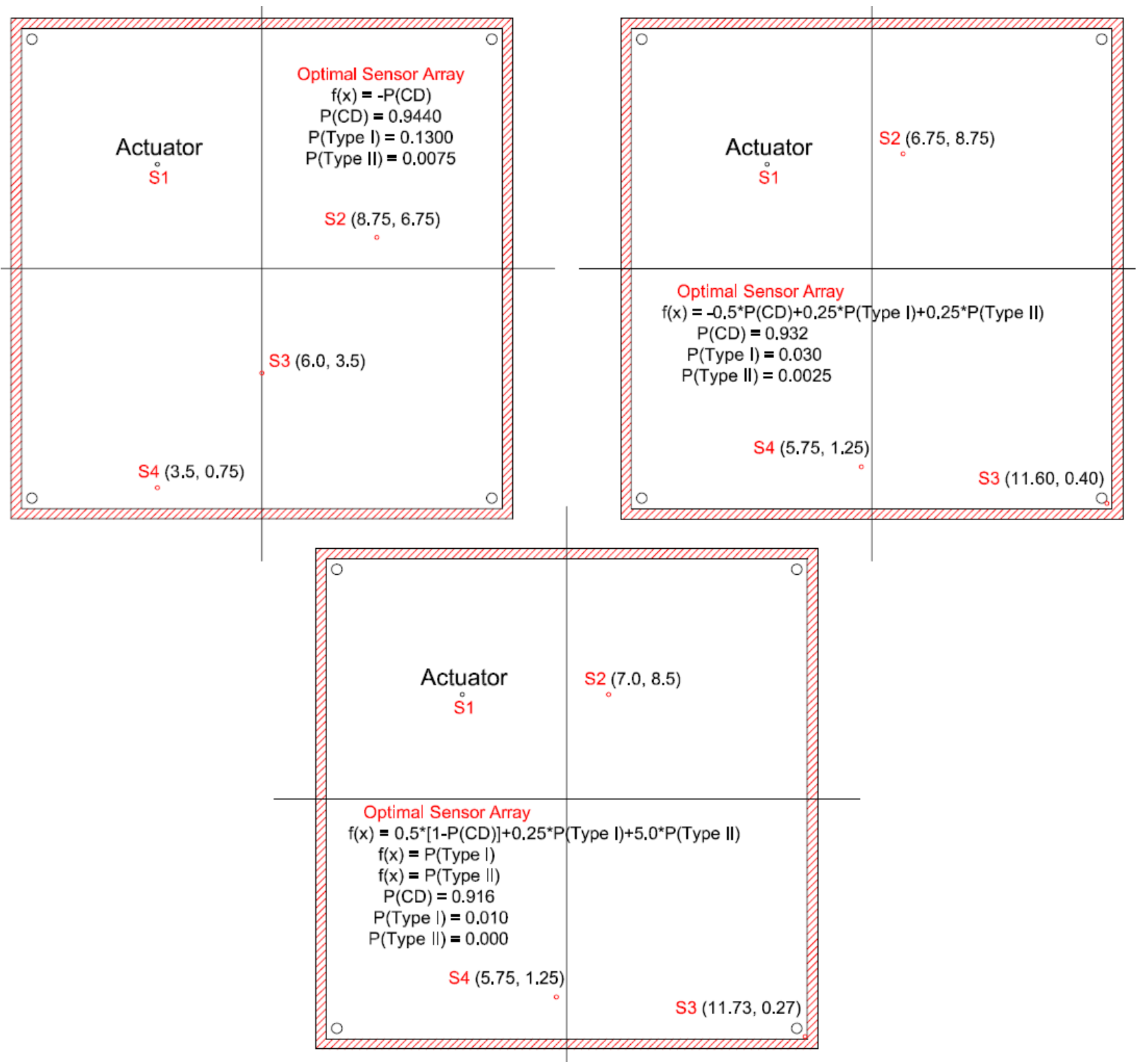

Figure 6. Results: optimal sensor arrays corresponding to different objective functions.

Difficulties were observed while optimizing the $3^{\text {rd }}$ objective function, $f(x)=-P($ TypeII $)$. Several sensor configurations produced probabilities of missed detection of zero percent, creating many optimal solutions, since a probability of missed detection less than zero percent is not possible. The authors utilized the additional information gathered during the optimization of objective functions 2, 4, and 5, and declared $\{S 2(7.0,8.5) ; S 3(11.73,0.27) ; S 4(5.75,1.25)\}$ to also be the optimal solution for the $3^{\text {rd }}$ objective function. 


\section{Conclusion}

A methodology for sensor placement optimization under uncertainty is developed in this paper. The method consists of four components: (1) structural simulation and model validation, (2) probabilistic analysis, (3) damage detection, and (4) sensor placement optimization. The methodology is applied to the optimization of the sensor array of a SHM system for a simplified TPS component.

Further work is required in regards to validating this methodology with experimental data. An additional investigation is necessary to determine the optimum number of sensors. In this paper the number of sensors was fixed; only their coordinates were design variables. It is reasonable to assume that as the number of sensors distributed across the structure increases, the estimated SHM performance measures improve. However, due to weight penalties associated with additional sensors, as well as complexity constraints with respect to the amount of data acquired by the sensing system that requires processing (and therefore processing power), the number of sensors applied to the structure must be minimum. In addition, future work needs to incorporate sensor reliability and redundancy into the optimization. Issues such as sensor performance and failure under various environmental and operating conditions need to be addressed.

\section{Acknowledgments}

This research is sponsored by the United States Air Force Research Laboratory (project monitor: Mark Derriso) through subcontract to Anteon Corporation. The authors gratefully acknowledge this support. The authors also gratefully acknowledge valuable discussions with Dr. Steven Olson at University of Dayton Research Institute and Dr. Martin DeSimio at Alliant Techsystems, Inc., in the development of the proposed methodology and their help with finite element models and model validation experiments.

\section{References}

${ }^{1}$ Shkarayev, S., Krashantisa, R., and A. Tessler, "An Inverse Interpolation Method Utilizing In-Flight Strain Measurements for Determining Loads and Structural Response of Aerospace Vehicles." in proceedings of $3^{\text {rd }}$ International Workshop on Structural Health Monitoring, Stanford, CA, September 12-14, 2001.

2 Hiramoto, K., Doki, H., and G. Obinata, "Optimal sensor/actuator placement for active vibration control using explicit solution of algebraic Riccati equation." Journal of Sound Vibration, Vol. 229, pp. 1057-75, 2000.

3 Abdullah, M., Richardson, A., and J. Hanif, "Placement of sensor/actuators on civil structures using genetic algorithms." Earthquake Engineering \& Structural Dynamics, Vol. 30, pp. 1167-84, 2001.

4 Yan, Y.J., and L.H. Yam, "Optimal design of number and locations of actuators in active vibration control of a space truss.” Smart Materials \& Structures, Vol. 11, pp. 496-503, 2002.

5 Simpson, M.T., and C.H. Hansen, "Use of genetic algorithms to optimize vibration actuator placement or active control of harmonic interior noise in cylinder with floor structure." Journal of Noise Control Engineering, Vol. 44, pp. 169-84, 1996. 
${ }^{6}$ Peng, F., "Actuator Placement Optimization and Adaptive Vibration Control of Plate Smart Structures." Journal of Intelligent Material Systems and Structures, Vol. 16, No. 3, pp. 263-71, 2005.

7 Demetriou, M.A., "Integrated Actuator-Sensor Placement and Hybrid Controler Design of Flexible Structures Under Worst Case Spatiotemporal Disturbance Variations." Journal of Intelligent Material Systems and Structures, Vol. 15, No. 12, pp. 901-21, 2004.

${ }^{8}$ Guo, H.Y., Zhang, L., Zhang, L.L., and J.X. Zhou, "Optimal placement of sensors for structural health monitoring using improved genetic algorithms.” Smart Materials \& Structures, Vol. 13, pp. 528-534, 2004.

9 Spanache, S., Escobet, T., and L. Trave-Massuyes, "Sensor Placement Optimization Using Genetic Algorithms." in proceedings of $15^{\text {th }}$ International Workshop on Principles of Diagnosis, Carcassonne, France, June 23-25, 2004

10 Berry, J.W., Hart, W.E., Phillips, C.A., Uber, J.G., and J.P. Watson, "Sensor Placement in Municipal Water Networks with Temporal Integer Programming Models." Journal of Water Resources Planning and Management, Vol. 132, No. 4, pp. 218-24, 2006.

11 Berry, J.W., Hart, W.E., Phillips, C.A., and J.P. Watson, "A Facility Location Approach to Sensor Placement Optimization." in proceedings of $8^{\text {th }}$ Annual Symposium on Water Distribution Systems Analysis, Cincinnati, $\mathrm{OH}$, August 27-30, 2006.

12 Watson, J.P., Hart, W.E., and R. Murray, "Formulation and optimization of robust sensor placement problems for contaminant warning systems." in proceedings of $8^{\text {th }}$ Annual Symposium on Water Distribution Systems Analysis, Cincinnati, OH, August 27-30, 2006.

${ }^{13}$ Berry, J.W., Fleischer, L., Hart, W.E., and C.A. Phillips, "Sensor placement in municipal water networks." in proceedings of The World Water and Environment Resources Congress, Philadelphia, PA, June 23-26, 2003.

14 Kessler, A., Ostfeld, A., and G. Sinai, "Detecting accidental contaminations in municipal water networks." Journal of Water Resources Planning and Management, Vol. 124, No. 4, pp. 192-8, 1998.

${ }^{15}$ Kumar, A., Kansal, M.L., and G. Arora, "Discussion of Detecting Accidental Contaminations in Municipal Water Networks." Journal of Water Resources Planning and Management, Vol. 124, No. 4, pp. 308-10, 1998.

${ }^{16}$ Watson, J.P., Greenberg, H.J., and W.E. Hart, "A Multiple-Objective Analysis of Sensor Placement Optimization in Water Networks." in proceedings of The World Water and Environmental Resources Congress, Salt Lake City, UT, June 27-July 1, 2004.

17 Guestrin, C., "Placement, Coordination and Tasking of Static and Mobile Sensor Networks." online at www.cs.ucsb.edu/ suri/Workshop06/guestrin.pdf accessed on September 10, 2006.

18 Ganesan, D., Cristescu, R., and B. Beferull-Lozano, "Power-Efficient Sensor Placement and Transmission Structure for Data Gathering under Distortion Constraints." in proceedings of ACM Transactions on Sensor Networks, Vol. 2, No. 2, pp. 155-81, May 2006.

19 Krause, A., Gupta, A., Guestrin, C., and J. Kleinberg, "Near-optimal Sensor Placement: Maximizing Information while Minimizing Communication Cost." in proceedings of $5^{\text {th }}$ International Conference on Information Processing in Sensor Networks, Nashville, TN, April 19-21, 2006.

20 Jourdan, D.B., and O.L. de Weck, "Layout Optimization for a Wireless Sensor Network Using a Multi-Objective Genetic Algorithm." in proceedings of IEEE's $60^{\text {th }}$ Vehicular Technology Conference, Los Angeles, CA, September 26-29, 2004.

${ }^{21} \mathrm{Li}$, D., Li, H., and C.P. Fritzen, "A New Sensor Placement Algorithm in Structural Health Monitoring." in proceedings of $3^{\text {rd }}$ European Workshop on Structural Health Monitoring, Granada, Spain, July 5-7, 2006.

22 Gao, H., and J.L. Rose, "Ultrasonic Sensor Placement Optimization in Structural Health Monitoring Using Evolutionary Strategy." in proceedings to AIP Conference on Quantitative Nondestructive Evaluation, Vol. 820, pp. 1687-93, March 6, 2006.

${ }^{23}$ Gao, H., and J.L. Rose, "Sensor placement optimization in structural health monitoring using genetic and evolutionary algorithms." in proceedings of SPIE Smart Structures and Materials, San Diego, CA, February 26, 2006.

${ }^{24}$ Dhillon, S.S., Chakrabarty, K., and S.S. Iyengar, "Sensor placement for grid coverage under imprecise detection." in proceedings of International Conference on Information Fusion, Annapolis, MD, July 7-11, 2002.

25 Parker, D.L., and W.G. Frazier, "Experimental Validation of Optimal Sensor Placement Algorithms for Structural Health Monitoring." in proceedings of $3^{\text {rd }}$ European Workshop on Structural Health Monitoring, Granada, Spain, July 5-7, 2006.

${ }^{26}$ Edwins, D.J., Modal Testing: Theory, Practice and Application. $2^{\text {nd }}$ Ed., Research Studies Press LTD., Philadephia, PA, 2000. 
${ }^{27}$ Rebba, R., and S. Mahadevan, "Probabilistic Assessment of CAE Models." in proceedings of SAE 2006 World Congress, paper no. 2006-01-0226, Detroit, MI, April 3-6, 2006.

${ }^{28}$ Haldar, A., and S. Mahadevan, Reliability Assessment Using Stochastic Finite Element Analysis. John Wiley \& Sons, Inc., New York, 2000.

${ }^{29}$ Shuping, H., "Simulation of Random Processes Using Karhunen-Loeve Expansion." The National University of Singapore, 2001.

${ }^{30}$ Tedesco, J.W., McDougal, W.G., and C.A. Ross, Structural Dynamics: Theory and Application. Addison Wesley Longman, Inc., Menlo Park, CA, 1999.

31 Sakamoto, S., and R. Ghanem, "Polynomial Chaos Decomposition for the Simulation of Non-Gaussian Nonstationary Stochastic Processes." Journal of Engineering Mechanics, pp. 190-201, February 2002.

${ }^{32}$ Deodatis, G., and R.C. Micaletti, "Simulation of Highly Skewed Non-Gaussian Stochastic Processes." Journal of Engineering Mechanics, pp. 1284-95, December 2001.

33 Corbin, M., Hera, A., and Z. Hou, "Location Damage Regions Using Wavelet Approach." online at http://wusceel.cive.wustl.edu/asce.shm/pdfs/corbin hera hou.pdf. accessed on October 29, 2003.

${ }^{34} \mathrm{Au}$, S.K., Yuen, K.V., and J.L. Beck, "Two-Stage System Identification Results for Benchmark Structure." online at http://wusceel.cive.wustl.edu/asce.shm/pdfs/au yuen beck.pdf. accessed on October 29, 2003.

${ }^{35}$ Banks, H.T., Joyner, M.L., Bincheski, B., and W.P. Winfree, "Real time computational algorithms for eddycurrent-based damage detection." Institute of Physics Publishing, Vol. 18, pp. 795-823, 2002.

${ }^{36}$ Doebling, S.W., Farrar, C.R., Prime, M.B., and D.W. Shevitz, Damage Identification and Health Monitoring of Structures and Mechanical Systems from Changes in Their Vibration Characteristics: A Literature Review. Los Alamos National Laboratory, Los Alamos, 1996.

${ }^{37}$ Duda, R.O., Hart, P.E., and D.G. Stork, Pattern Classification. $2^{\text {nd }}$ Edition, John Wiley \& Sons, Inc., New York, 2001.

${ }^{38}$ DeSimio, M., Miller, I., Derriso, M., Brown, K., and M. Baker, "Structural Health Monitoring Experiments with a Canonical Element of an Aerospace Vehicle." in proceedings of 2003 IEEE Aerospace Conference, Big Sky, Montana, March 8-15, 2003.

${ }^{39}$ Padula, L.S., and R.K. Kincaid, "Optimization Strategies for Sensor and Actuator Placement." NASA/TM-1999209126, April 1999.

${ }^{40}$ Padula, L.S., and R.K. Kincaid, "Optimal Sensor/Actuator Locations for Active Structural Acoustic Control." in proceedings of $39^{\text {th }}$ AIAA/ASME/ASCE/AHS/ASC Structures, Dynamics and Materials Conference, Long Beach, CA, April 20-23, 1998.

${ }^{41}$ Padula, L.S., and R.K. Kincaid, “Aerospace Applications of Integer and Combinatorial Optimization.” NASA TM-110210, October 1995.

${ }^{42}$ Raich, A.M., and T.R. Liszkai, "Multi-Objective Genetic Algorithm Methodology for Optimizing Sensor Layouts to Enhance Structural Damage Detection." in proceedings of $4^{\text {th }}$ International Workshop on Structural Health Monitoring, Stanford, CA, September 15-17, 2003.

${ }^{43}$ Spall, J.C., "An Overview of the Simultaneous Perturbation Method for Efficient Optimization." Johns Hopkins APL Technical Digest. Vol. 19, No. 4, pp. 482-92, 1998.

${ }^{44}$ Huyer W., and A. Neumaier, "SNOBFIT - Stable Noisy Optimization by Branch and Fit." submitted for review to ACM Transactions on Mathematical Software, online at http://www.mat.univie.ac.at/ neum/software/snobfit/. accessed on May 25, 2004.

${ }^{45}$ Olson, S., DeSimio, M., and M. Derriso, "Fastener Damage Estimation in a Square Aluminum Plate." submitted to Structural Health Monitoring Journal, 13 May, 2005.

${ }^{46}$ Ansys, ANSYS Release 9.0 Documentation. ANSYS, Inc., 2004.

${ }^{47}$ Guratzsch, R.F., Mahadevan, S., Olson, S., DeSimio, M., and M. Derriso. "Metrics for Validation of Modal Analysis Under Uncertainty." submitted for review to Journal of Vibration and Control, February 20, 2006.

48 Shinozuka, M., and Deodatis, G., "Simulation of stochastic processes by spectral representation" Applied Mechanics Review, Vol. 44, No. 4, pp. 191-204, 1991.

${ }^{49}$ VanMarcke, E., Random Fields: Analysis and Synthesis. The MIT Press, Cambridge, MA, 1983.

${ }^{50}$ The Royal Aeronautical Society, Material Properties Handbook: Volume I Aluminum Alloys. North Atlantic Treaty Organization Advisory Group for Aerospace Research and Development, London, with Amendments from 1959 and 1966.

${ }^{51}$ Oppenheim, A.V., and R.W. Schafer. Digital Signal Processing, Prentice-Hall, Englewood Cliffs, NJ, 1975. 
${ }^{52}$ Welch, P.D., "The Use of Fast Fourier Transform for the Estimates of Power Spectra: A Method Based on Time Averaging Over Short, Modified Periodograms." IEEE Transactions on Audio Electroacoustics, Vol. AU-15, pp. 70-3, June 1967.

${ }^{53}$ Somol, P., Pudil, P., Novovicova, J., and P. Paclik, “Adaptive Floating Search Methods in Feature Selection.” Pattern Recognition Letters, Vol. 20, pp. 1159-63, 1999.

${ }^{54}$ Jain, A., and D., Zongker, "Feature Selection: Evaluation, application and small sample performance." IEEE Transactions on PAMI, Vol. 19, pp. 153-8, 1997.

${ }^{55}$ Guratzsch, R.F., and S. Mahadevan, "Feature Extraction and Selection Algorithms for Structural Damage Detection." submitted for review to Structural Control \& Health Monitoring, June 27, 2006.

${ }^{56}$ Kohavi, R. and F. Provost, "Glossary of Terms." Machine Learning, Vol. 30, No. 2/3, pp. 271-4, 1998.

${ }^{57}$ The MathWorks, Inc., MATLAB Version 7.0.4.365 (R14) Service Pack 2, January 29, 2005. 